\title{
EFFECTS OF USER BEHAVIOUR ON GSM AIR INTERFACE PERFORMANCE
}

\author{
K. Diawuo ${ }^{1}$ and B. P. Adih ${ }^{2}$ \\ ${ }^{I}$ Dept. of Computer Engineering, Kwame Nkrumah University of Science and Technology \\ ${ }^{2}$ MTN GHANA
}

\begin{abstract}
The behaviour of user when accessing the services offered by the GSM network has an impact on the performance of the network particularly at the air interface. A good understanding of user behavior and its impact on network performance is therefore of paramount concern to the network planning engineer. In this paper a model is developed based on the multi-agent paradigm for the study of user behavior and its impact on the blocking probability at the air interface. Aspects of user behavior studied are the flashing phenomenon and the effects of variations in mean call duration for a given traffic load per user. The effect of encouraging users to modify their behaviour through feedback under high load situations is also studied. The study revealed that, making modifications to the tariffs by encouraging longer talk time in preference to several short calls or flashing and the use of feedback to customers did not only improve perceived but also real network performance.
\end{abstract}

Keywords: Air interface, multiagent, flashing

\section{INTRODUCTION}

The number of GSM subscribers is constantly increasing as well as the number of service providers. In such an environment operators continuously try to improve their network coverage, the variety of services they offer and the quality of their services both in real and perceived terms. These may effectively be achieved by paying attention to both the quantum of traffic a network is expected to handle and the nature of the traffic. Telecommunications traffics can be modeled based on Figure 1 (Ericsson, 2003).

Due to the random nature of the offered traffic, it may on occasion exceed the baseline used in network dimensioning. This may lead to a significant degradation in service level; this situation is amplified in mobile networks where users carry their terminals along with them.

Attempting to design a network to accommodate situations such as these would lead to over dimensioning and wastage of resources. Instead, a novel approach would be to try and control the fraction of traffic demand that is offered to the network. This could be done by monitoring the offered traffic and giving feedback to users when the network capacity is at or nearing full utilization. Such method is particu- 


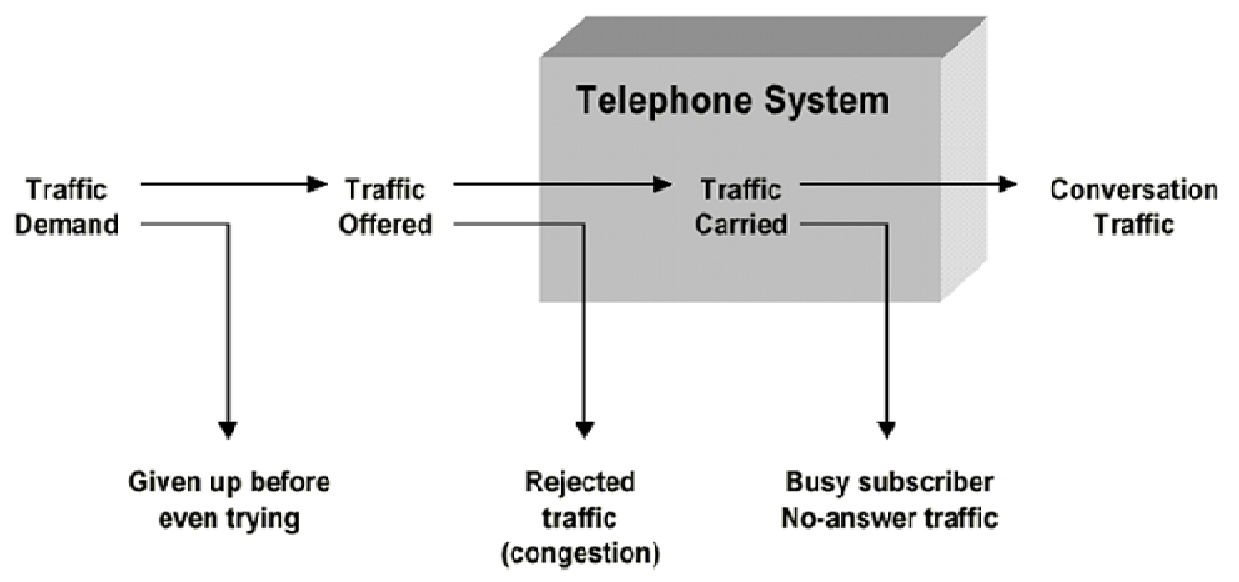

Fig. 1: Components of Traffic

larly suited for cellular networks where such traffic hotspots tend to be much localized. Mechanisms such as cell broadcast exist in the GSM specifications for providing the requisite feedback and suggesting methods to users to control the proportion of the traffic demand that turns into offered traffic. This mode of traffic control has been investigated by Shinagawa et al. (2009), where it was pointed out that the feedback also tends to boost satisfaction level of customers.

The queue based models that were hither used for the analysis of telephony systems have become increasingly inadequate for the study of modern telephone system including cellular networks. The number of tools available to the researcher has also increased in recent times with one of the more interesting developments being Multiagent simulations (MAS). These have gained prominence as effective tools in the study of systems involving large numbers of autonomous agents. Multiagent simulations have been applied successfully in study of various systems in sociology, biology, physics, economics, and marketing (Center for Connected Learning 2009) and in real time dynamic applications (Fan et al., 2008 and Wu et al., 2010). Multiagent simulation systems hold promise as a means for the study of teletraffic models that use the traffic source approach (Tutschku et al., 1998). Shinagawa et al. (2009), however were the only ones found in the literature to have used this approach in the study of teletraffic.

In this paper a model is developed using the MAS paradigm to study the effect of some aspects of user behavior on network performance. The effectiveness of feedback as the means of controlling traffic demand during periods of high network utilization is also studied.

\section{MODEL DESCRIPTION}

A model in MAS consists of series of incorporated software objects, called agents (Wooldridge, 2002). The agent representing the 'individual' is programmed to be autonomous and can communicate with its environment and other agents. Macal and North (2006) provide a general outline for steps required in developing a MAS simulation. Siebers and Aickelin (2007) went further by suggesting state charts as a means of representing the internal processes of agents. The central state in the state chart is the idle start which links to all state that are not dependent on a specific order of execution. State transitions occur by means of triggers which may be in the form of rules, formulae or procedures. The development environment used is called Netlogo (Center for Connected 
Learning 2009) while the simulation environment is made up of two types of agents: the callers and the base stations.

\section{Callers}

For the caller agent model, the user behaviour was broken down into two distinct components, traffic generation and movement pattern. A description of the models for these two facets is given in Figure 2 which represents the various states the callers go through as they generate traffic (Onur et al., 2000) Callers are normally in the idle state and they leave this state by generating call requests. A request may be granted by the associated base station at which time the caller then goes into the ring state. This state allows the model to capture the situation in which a user after receiving the ringing tone breaks the connection, a phenomenon known as "flashing." Mehotra (1997) and Onur et al. (2000) suggested that the GSM specifications mandate a number of automatic retrials when the initial call attempt fails.

The maximum number of retrials is however, set by the network operator. Thus, if the initial call attempts fail, these automatic retrials take place. If these also fail the user may manually redial in order to establish a connection.

After the ringing period the user goes into the speech state. The caller remains in this state for some time and then returns to the idle state. Due to possible movement of the caller during the connection period it may become necessary to change base station (handover). If the initial

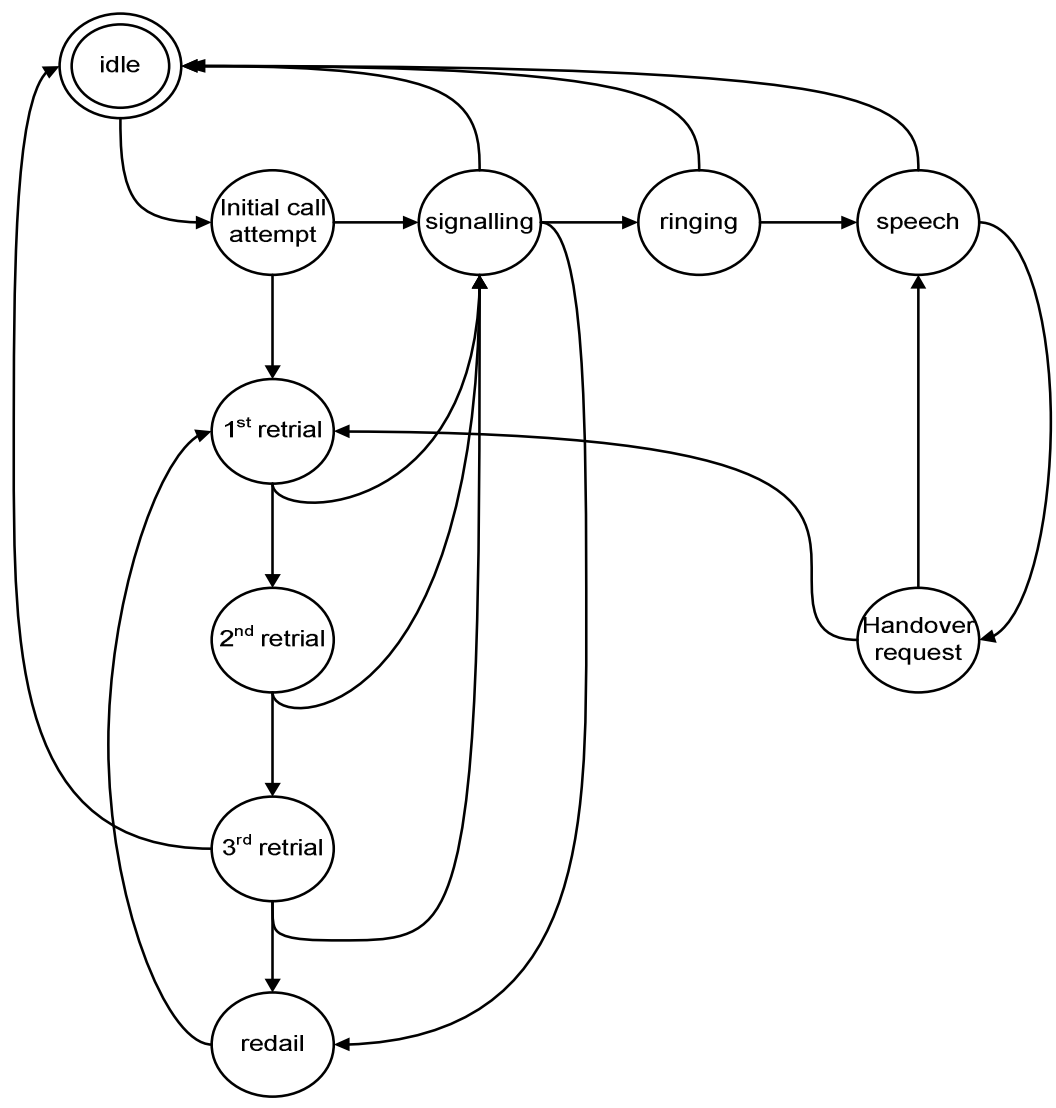

Fig. 2: Model of Callers States and Transitions 


\section{Diawuo and Adih}

handover attempt fails a number of automatic request are made but if these also fail the line is dropped. In this case also the user may redial in an attempt to re-establish a connection.

The simulation assumes that the caller is located in a commercial urban environment where most callers are assumed to be walking and those using vehicular transport are also moving at relatively low speeds. The average walking speed of an adult human is around $5 \mathrm{~km}$ per hour (Wikipedia 2009). The maximum stipulated speed for a vehicle in the urban environment is $50 \mathrm{~km}$ per hour. This maximum speed is however not attained in the scenario that is used in the simulations. Given this background the speed of callers is uniformly distributed between $0-25 \mathrm{~km}$ per hour. After each simulation cycle the user chooses a new direction in a cone $40^{\circ}$ wide in the direction of motion. This ensures that there is correlation in the direction of movement of the user.

The simulation area is of finite dimension. Davies (2000) and Bettstetter (2001) gave a method of dealing with callers that go outside the bounds of the simulations areas: a caller that falls off one edge appears on the opposite edge. Every time there is a change of position, the caller recalculates its distance relative to all the base stations and associates itself with the nearest one. If the caller is active and the nearest base station is different from its current base station, a handover occurs. When a caller in the automatic retrial state undergoes a handover, the call is dropped. The caller treats this event as a failed call attempt. A total of 2000 caller agents were used in the simulations.

\section{Base Station Agents}

These agents play the role of base station in actual GSM networks. When a caller agent requests a traffic channel, the base station checks the number of callers associated with it that are currently active, if it is less than the number of available traffic channels, the caller is allowed to change into the active state. Kivi (2006) gave an overview of how service utilization can be measured in wireless data network. The approaches that were suggested can be adapted for GSM networks. In our model the base stations are responsible for monitoring the utilisation of their traffic channels. When the average number of active callers within a specified period exceeds a preset threshold, the base station broadcasts a message encouraging callers associated with it to refrain from generating additional traffic. In real networks such a procedure could be implemented using cell broadcast. A total of 7 base station agents were used in each simulation. One was located at the centre of the simulation area and other six were arranged in a regular hexagonal pattern around it.

\section{Air Interface Simulation}

In the model only one type of traffic channel was used in the simulation as opposed to the full rate and half rate channels that exist in the GSM specifications. In addition, signalling channels were introduced into the simulation so as to realise the functions of the Standalone Dedicated Control Channels (SDCCH). In all, three sets of simulations were performed. In the first set, callers generated traffic based on the call generation model. In the second set of simulations, callers used a combination of signalling channel (SMS/USSD) only; and signalling and traffic channels when their associated base stations were congested. In the final set, callers increased the amount of time they spent in the idle state when prompted by the associated base station. At the end of each individual simulation the sum total of requests from users for signalling and traffics channels along with the number of requests that were successful were logged. The average blocking probability $\mathrm{P}_{\mathrm{b}}$ at the air interface was used as the metric for measuring performance. The blocking probability was estimated from the results using equations 1 to 5 .

$$
P_{S}=\frac{N 1}{N 2}
$$


$P_{t}=\frac{N 3}{N 4}$

$P_{b s}=1-P_{s}$

$P_{b t}=1-P_{t}$

$P_{b}=1-P_{t} P_{s}$

Where: $N 1$ is the number of signal channel grants

$N 2$ is the number of signal channel requests

$N 3$ is the number of traffics channel grants

N4 is the number of traffics channel Requests

$\mathrm{P}_{\mathrm{s}}=$ Average probability of success in signalling channel allocation

$\mathrm{P}_{\mathrm{t}}=$ Average probability of success in traffic channel allocation

$\mathrm{P}_{\mathrm{bs}}=$ Average probability of blocking on the signalling channels
$\mathrm{P}_{\mathrm{bt}}=$ Average probability of blocking on the traffic channels

$\mathrm{P}_{\mathrm{b}}=$ Average overall probability of blocking at the air interface

\section{RESULTS AND DISCUSSION}

Effects of Variations in Mean Call Duration

The effect of variations in the mean call duration for a given level of channel utilization per user is analysed. Figure 3 indicates that for a given level of channel utilization per user, decreases in the mean call duration leads to an increase in the blocking probability. It can also be observed that the blocking probability increases with increasing utilization per user. It is again clear that reduction in call durations, leads to an increase in the call intensity. The results indicate that there has been an increase in the amount of traffic carried by the signalling channels even though the traffic on the traffic channels in terms of speech remains constant.

This increase causes a decrease in $P_{S}(1)$ which in turn leads to an increase in $\mathrm{P}_{\mathrm{b}}$ according to (5). The simulation result of the blocking probability on the signalling channels is shown in Table 1.

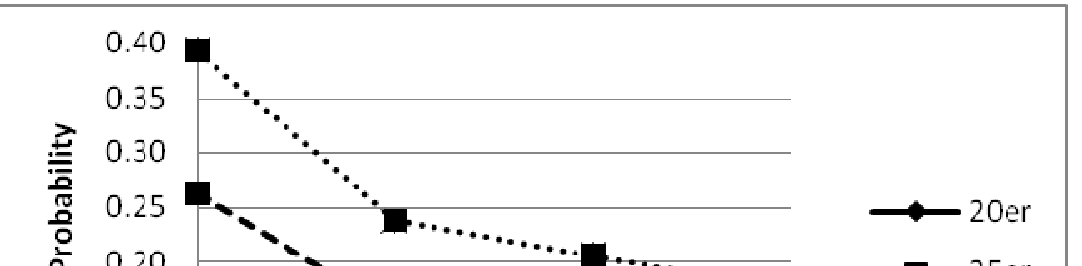


Table 1: Blocking Probability on Signalling Channels $\left(P_{b s}=1-P_{s}\right)$

\begin{tabular}{lllll}
\hline Mean Traffic Per User & & \multicolumn{2}{c}{ Mean Call Duration } & \\
& $\mathbf{3 0 ~ \mathbf { ~ s e c }}$ & $\mathbf{6 0 ~ \mathbf { ~ s e c }}$ & $\mathbf{9 0 ~ \mathbf { ~ e c }}$ & $\mathbf{1 2 0} \mathbf{~ s e c}$ \\
\hline 20 mErlang & 0.00181 & 0.00012 & 0.00027 & 0.00000 \\
25 mErlang & 0.00742 & 0.00049 & 0.00026 & 0.00000 \\
30 mErlang & 0.01546 & 0.00127 & 0.00026 & 0.00014 \\
35 mErlang & 0.03734 & 0.00411 & 0.00118 & 0.00038 \\
40 mErlang & 0.07107 & 0.00915 & 0.00244 & 0.00107 \\
\hline
\end{tabular}

The assignment of a traffic channel can be broken into two distinct parts. The first part is where the ringing tone is received and the second is where the actual conversation goes on. With increasing call intensity the proportion of time used for providing the ringing tone on the traffic channels increases. In the simulations the mean ringing tone duration was taken to be 15 seconds. With mean call duration of $30 \mathrm{sec}-$ onds, the additional traffic burden on the traffic channels for the ringing tone was $50 \%$ of that used for actual speech however, with mean call duration of 120 seconds, this value dropped to $12.5 \%$. Thus as mean call duration decreases the traffic carried on the traffic channels increases significantly in comparison to the actual speech carried.

\section{Effects of Flashing}

Figure 4 shows the effect of flashing on system performance. It shows the variation in blocking probability as the proportion of initial call attempts that are flashes increases for different levels of traffic per It can be seen from the graphs that irrespective of the traffic level per user the blocking probability increases as the proportion of flashes increases. The degradation in blocking probability is however not very pronounced for low levels of traffic per user. Flashes are actually calls with no speech component and thus place the same amount of signalling and ringing tone traffic burden on the network as actual calls involving speech.

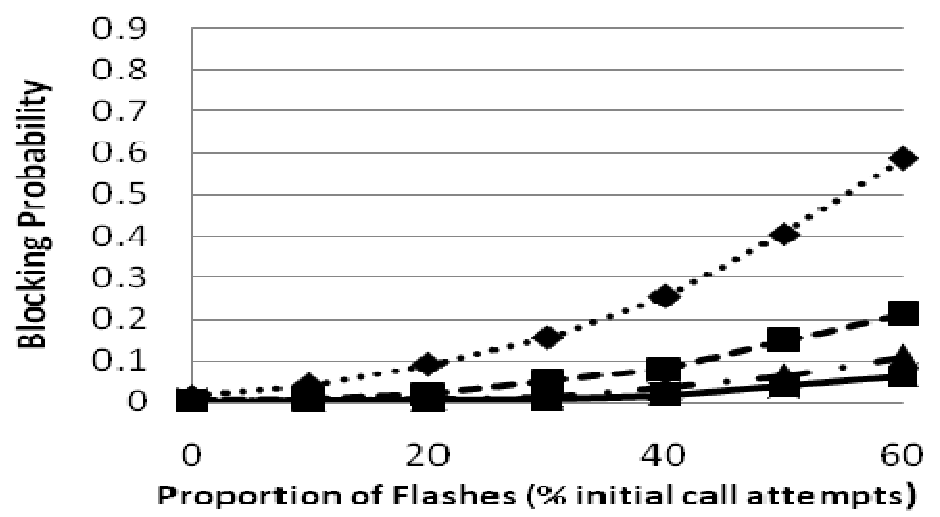

(a) 
User Behaviour on GSM Air Interface... 121

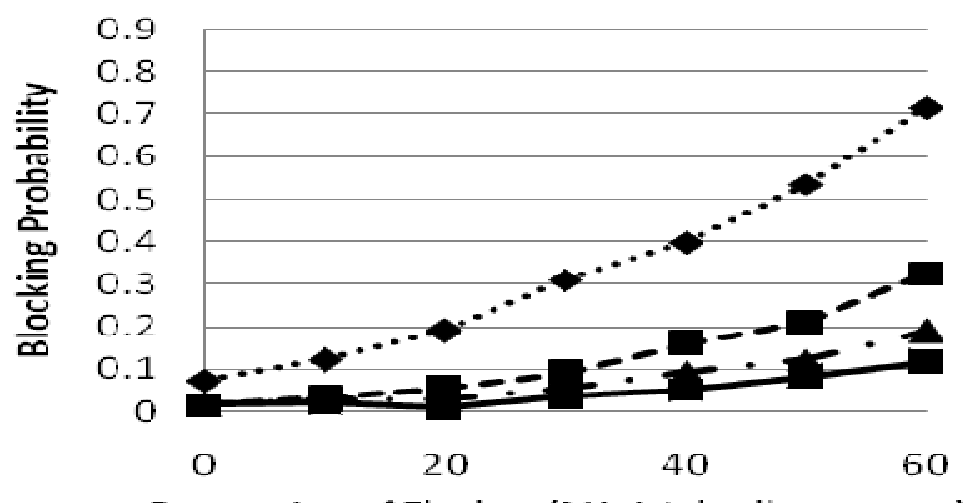

Proportion of Flashes (\% initial call attempts)

(b)

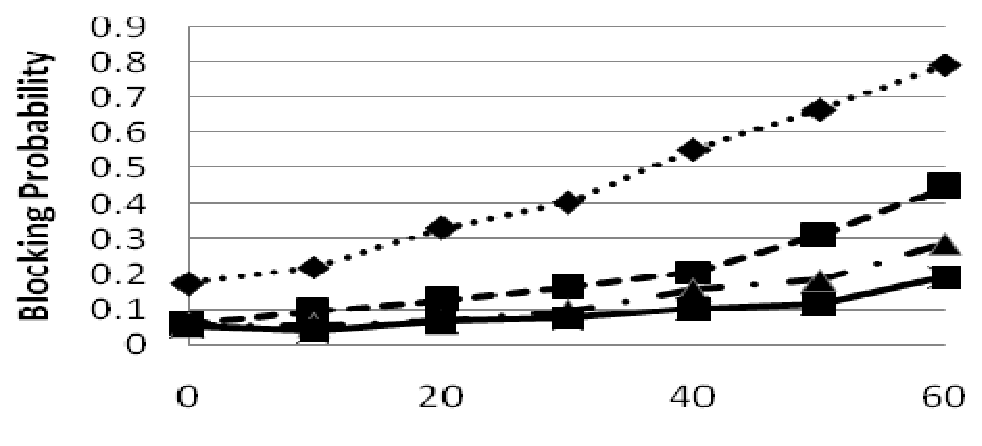

Proportion of Flashes (\% initial call attempts)

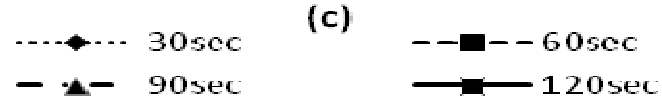


Modified

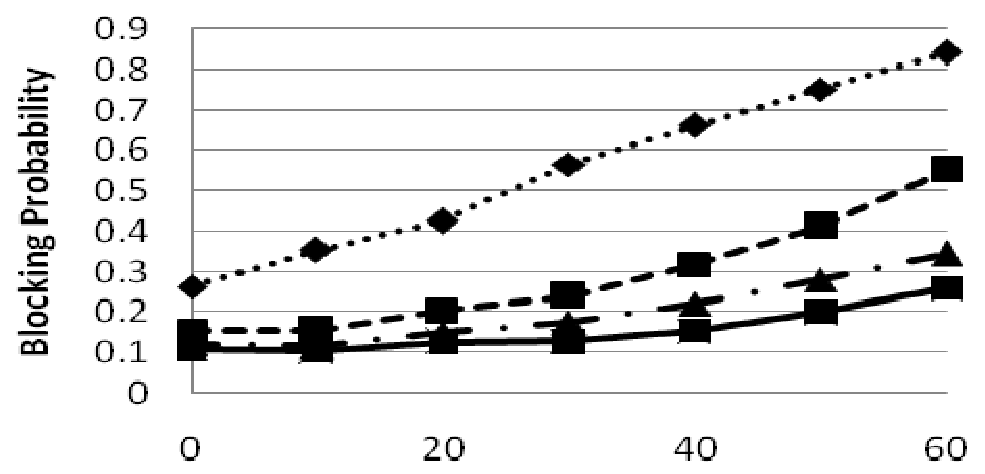

Proportion of Flashes (\% initial call attempts)

(d)

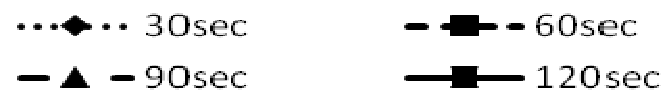

Fig. 4: Effects of Flashing in mErlang at (a) 20, (b) 25, (c) 30, (d) 35

\section{User Behaviour}

It is clear that user behaviour plays a significant role with regards to blocking probabilities at the air interface. This was studied using two modifications to user calling pattern as:

(a) The use of alternative channels present at the air interface for communication in stead of traffic channels (b) Postponement of the time of the call

In the first scenario users are assumed to use means such as SMS and USSD as an alternative to using voice when the network is congested. Figure 5 compares the blocking probabilities from the basic model with those obtained from the situation where users use SMS during congestion.

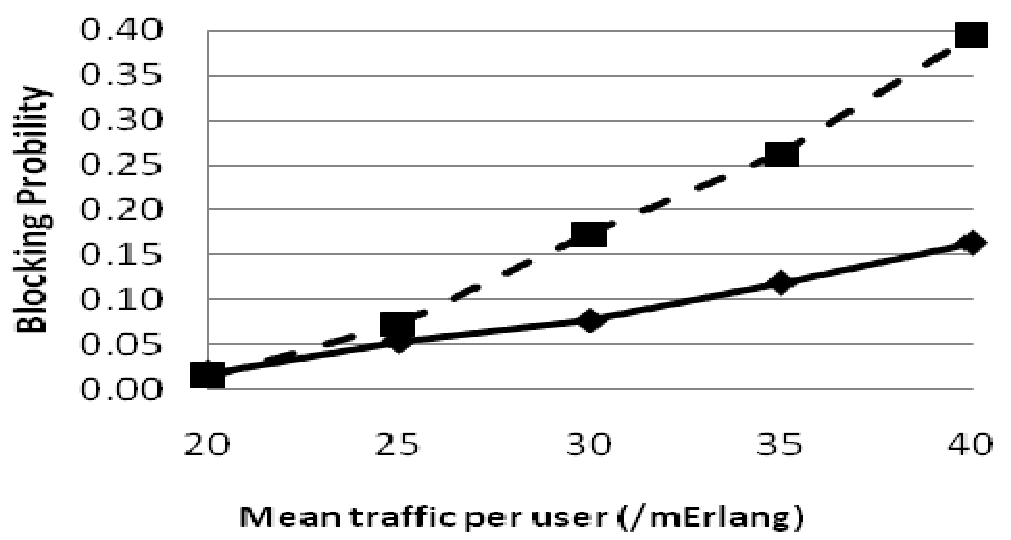

(a) 
User Behaviour on GSM Air Interface... 123

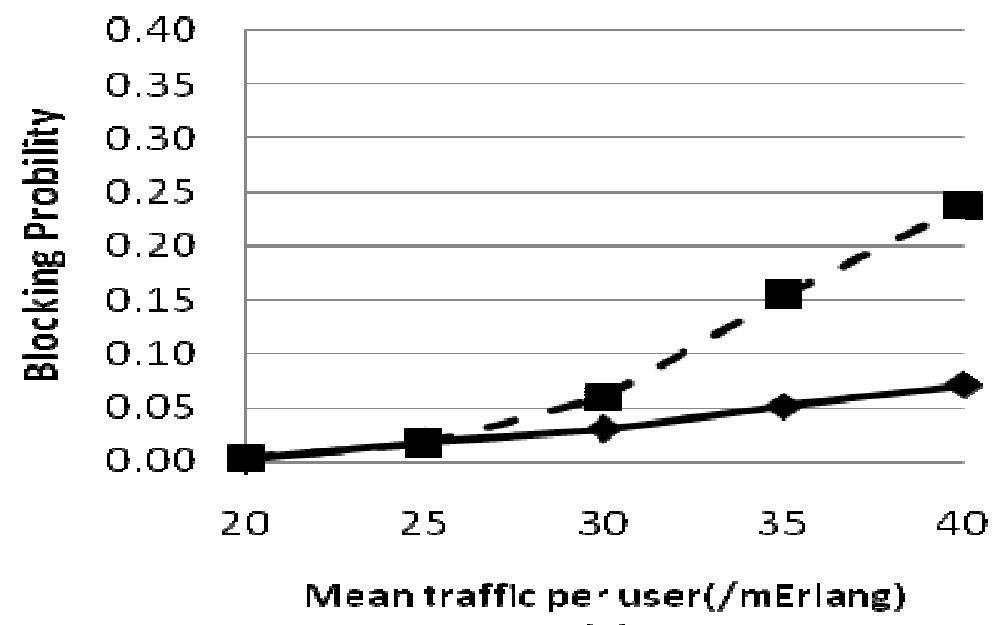

(o)

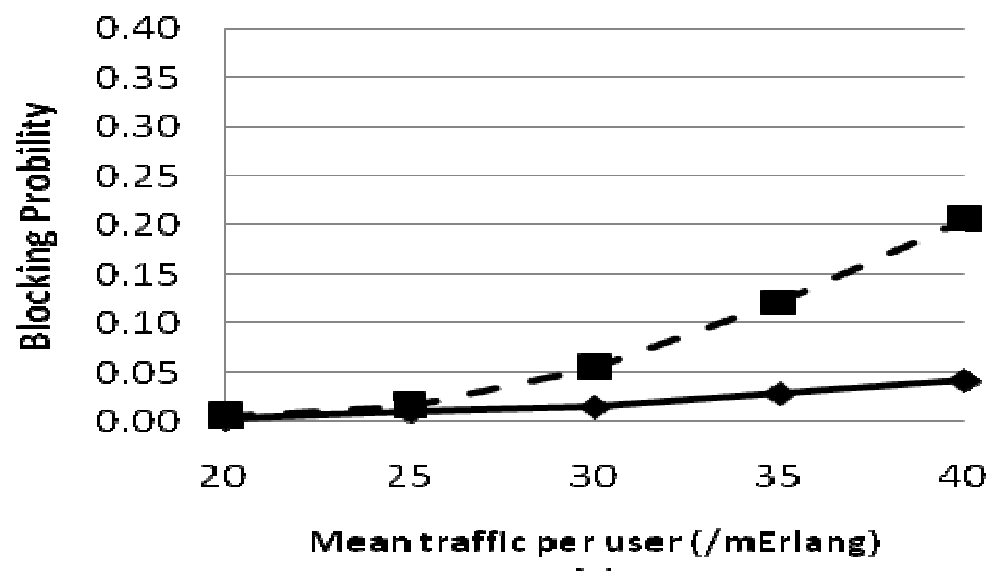

- E Basic

(c)

- Modified Behaviour (Alternate Channel) 


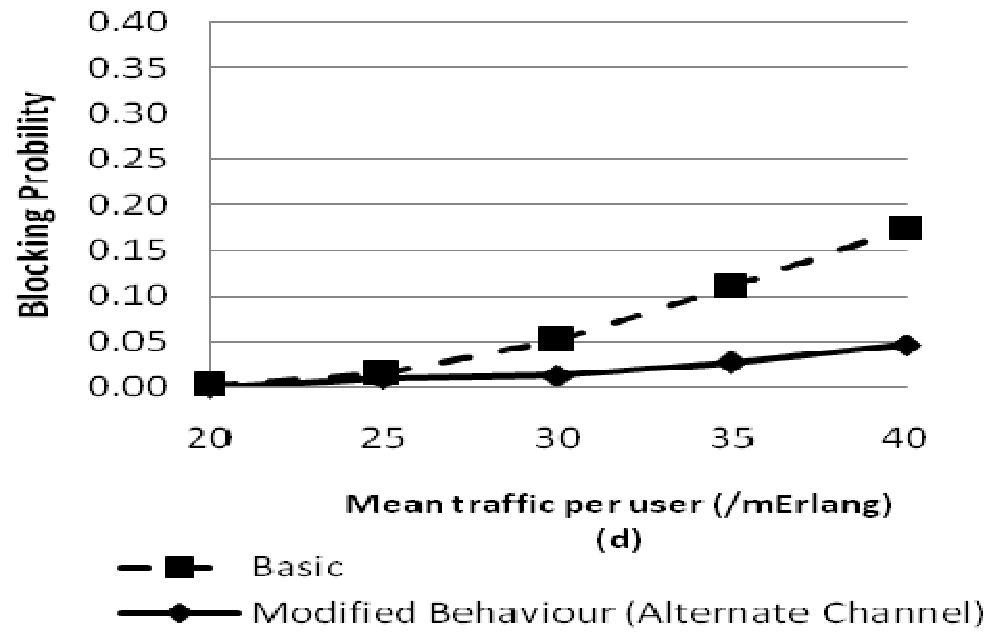

Fig. 5: Comparison of Blocking Probabilities - basic model and model with signalling channels as alternative (SMS), (a) 30sec, (b) 60sec, (c) 90sec (d) 120sec.

The individual graphs present the blocking probability with increasing traffic per user for different mean call durations. The results indicate that a significant improvement may be achieved by encouraging users to take advantage of the alternative means of communication over the air interface that are available aside the voice. This method seems to be particularly effective in situations where the blocking probability is high.

It can also be observed that callers may only be encouraged to use the alternative means of communication when congestion is encountered. Thus at low traffic levels where congestion is minimal (less than 25 mErlang) there is little difference between the basic and modified behaviour however, the overall blocking probability is greatly improved for higher traffic levels. The second modification of user behaviour studied is the deferment of the time at which the call is made. Figure 6 compares the results of the deferred call scenario with the basic case. The individual graphs present the blocking probability with increasing traffic per user for different mean call durations. Here also it can be seen that deferring calls during period of congestion can significantly decrease the probability of blocking at the air interface.

Figure 7 shows the comparison between the two behaviour modifications considered above with the basic model as reference. It is clear from all the four graphs that, irrespective of the mean call duration, deferment of calls provide the least blocking probability than the use of alternative modes of communication. This is in agreement with that reported in Shinagawa et al. (2009). In their study they found that using the alternative means available provided a better performance compared to deferring calls however, this study presented a reverse claim. The difference may arise from difference in the models of user behaviour used in both simulations. Unfortunately, detailed information on their model is unavailable for a thorough analysis and comparison. 


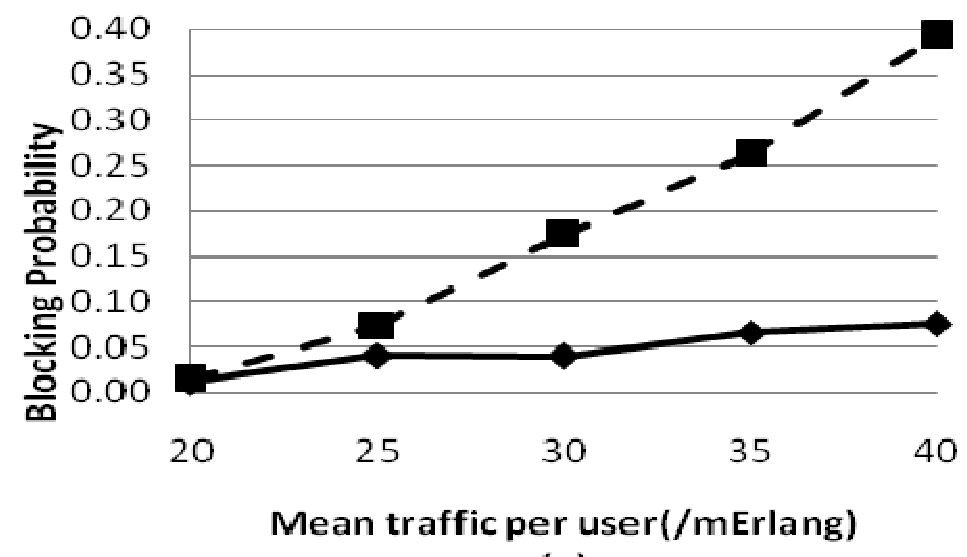

(a)

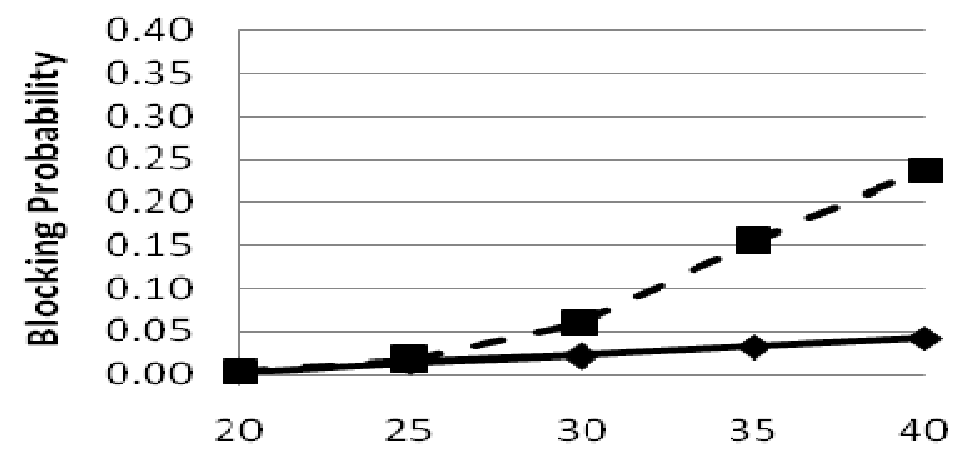

Mean traffic per user(/mErlang)

(b) 

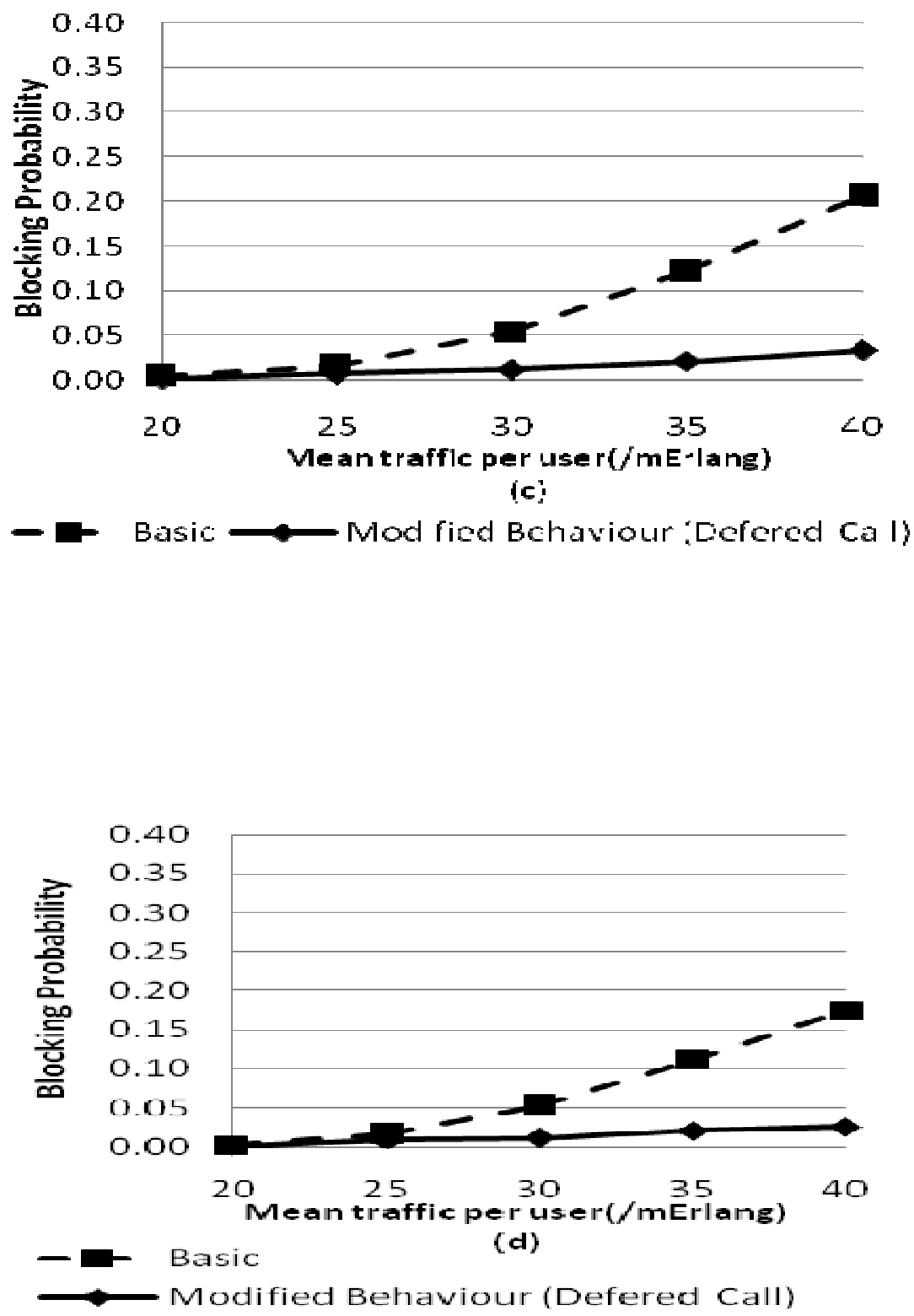

Fig. 6: Comparison of Blocking Probabilities - Basic behaviour and Call deferment. (a) 30sec, (b) 60sec, (c) 90sec, (d) $120 \mathrm{sec}$. 


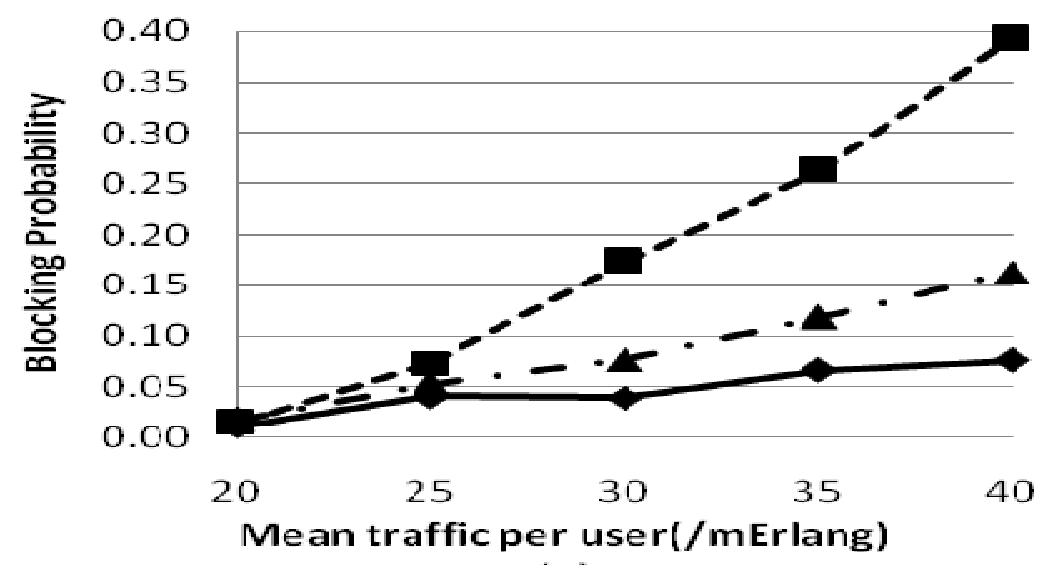

(a)

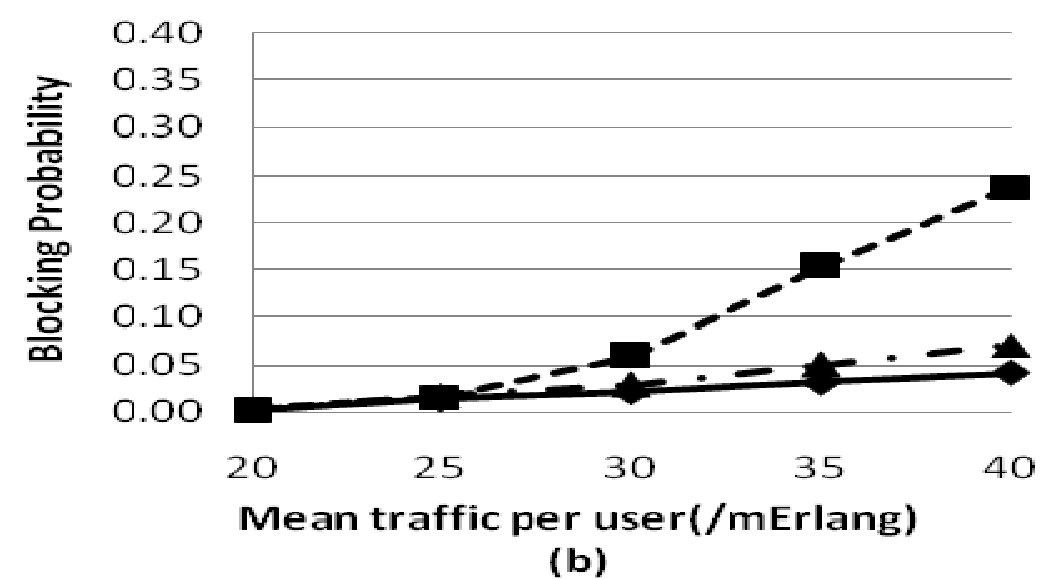

Journal of Science and Technology @ KNUST April 2011 

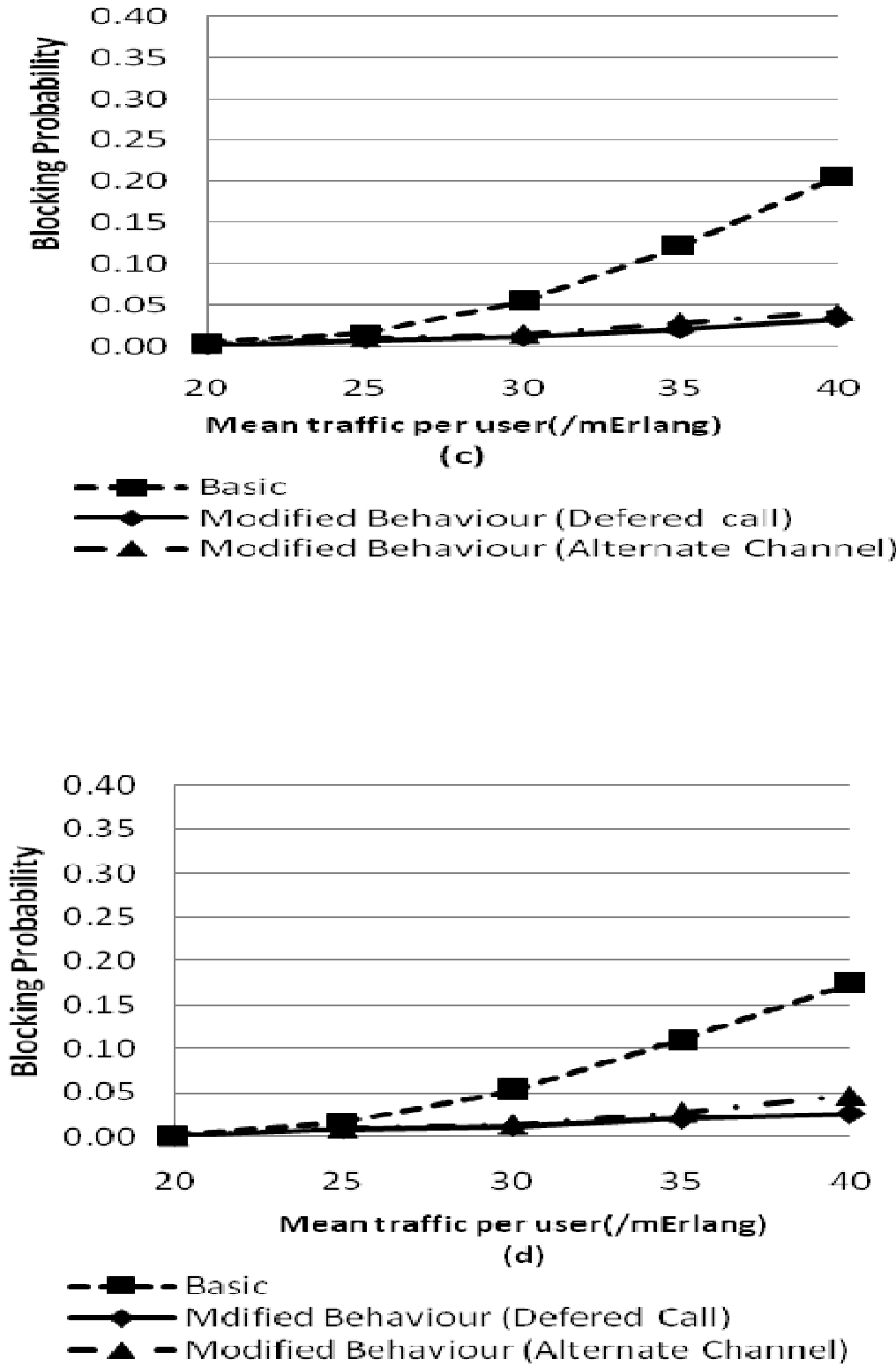

Fig. 7: Comparison of Blocking Probabilities - Basic behaviour, alternate channels and Call deferment. (a) 30sec, (b) 60sec, (c) 90sec, (d) 120sec. 


\section{CONCLUSIONS}

In this paper, it was found that both low mean call durations and a large number of repeated call attempts have a significant negative impact on air interface performance. Encouraging users to modify their call patterns gave significant improvements in air interface performance. Although these gains were not significant at low level of traffic per user but as traffic increased the improvements became more significant with up to $75 \%$ reduction in blocking probabilities.

From the study therefore, making modifications to the tariffs by encouraging longer talk time in preference to several short call or flashing and the use of feedback to customers will not only improve perceived but also real network performance.

\section{REFERENCES}

Bettstetter, C. (2001). Smooth is Better than Sharp: A Random Mobility Model for simulation of wireless networks in ACM MSWiM, Rome, Italy, July 2001.

Center for Connected Learning (2009). http:// ccl.northwestern.edu/netlogo/ accessed on 19th January 2009.

Davies, V. A. (2000). Evaluating mobility models within an ad hoc network, Master Thesis. Colorado School of Mines. Colorado, USA.

Ericsson A. B. (2003). APG40 Operation and Maintenance 2003.

Fan, C., and Chen, X. (2008) "Optimal Action Criterion and Algorithm Improvement of Real-Time Dynamic Program ming." Journal of Software,19 (11):2869-2878.

Kivi , A. (2006). Measuring Mobile Data Service Usage And Traffic In Mobile MultiAccess Networks, Networking Laboratory, Helsinki University of Technology.
Macal, C. M., North, M. J. (2006). Tutorial On Agent-Based Modelling And Simulation Part 2: How To Model With Agents in Proceedings of the 2006 Winter Simulation Conference, Center for Complex Adaptive Agent Systems Simulation (CAS2), Decision \& Information Sciences Division, Argonne National Laboratory, USA.

Onur, E., Delic, H., Ersov, C., Caglayan, M.U (2000). "On the retrial and redial phenomena in GSM networks". Proceedings of the IEEE Wireless Networking and Communications Conference. Vol. 2:885-889.

Shinagawa, N., Miura, A., Kaneda, S. and Akinaga, Y. (2009). Traffic Control By Influencing User Behavior available at http://www.nttdocomo.co.jp/english/ binary/pdf/corporate/technology/rd/ technical_journal/bn/vol _ 4/ vol7_4_34en.pdf accessed on19th January 2009 .

Sieber, P., and Aickelin, U. (2007). Introduction To Multi-Agent Simulation", School of Computer Science \& IT, University of Nottingham.http://eprints.notting ham.ac.uk/645/

Tutschku, K., Leskien, T. and Trangia, P. (1998). Spatial Traffic Estimation and Characterization For The Design Of Mo bile Communication Networks, IEEE Journal on Selected Areas of Communication -- Special Issue on Advances in Computational Aspects of Teletraffic Models, 16(5): 804-811.

Wikipedia (2009). http://en.wikipedia.org/wiki/ Walking

Wooldridge, M. (2002). An Introduction to Multiagent Systems. John Wiley and Sons Ltd, Chichester, England.

Wu, F., Zilberstein, S. and Chen, $\mathrm{X}$. 


\section{Diawuo and Adih}

(2010). “Trial-Based Dynamic Programming for Multi-Agent Planning," In: Proc. of the Twenty-Fourth AAAI Conference on Artificial Intelligence (AAAI 10), Atlanta, Georgia, USA,. July 11-15, 2010. 